\title{
Successful Treatment of LVAD Patient with Infection-Induced Pseudoaneurysm using Endovascular Stent Graft
}

\author{
Rustum S ${ }^{1 *}$, Schmitto $\mathbf{J}^{1}$, Dogan $\mathbf{G}^{1}$, Umminger $\mathbf{J}^{1}$, Haverich $\mathrm{A}^{1}$ and Wilhelmi $\mathbf{M}^{1,2}$
}

${ }^{1}$ Division of Vascular and Endovascular Surgery, Department of Cardiothoracic, Transplantation, and Vascular Surgery, Hannover Medical School, Hannover, Germany ${ }^{2}$ Center for Competence for Cardiovascular Implants, Hannover Medical School, Hannover, Germany

\begin{abstract}
Peudoaneurysms are rare complications related to left ventricular assist device (LVAD) implantation and mostly occur at the left ventricular apex, as well as the anastomosis of the outflow graft to the ascending aorta. Beside anastomosis related complications and an erosion of the graft with a sternal wire they can occur due to an infection. Nevertheless pseudoaneurysms represent a major complication potentially leading to significant morbidity and mortality and in most cases necessitating open-surgical management. Here we report on a case of an infectioninduced pseudoaneurysm in close proximity to the LAVD's outflow cannula which was treated successfully with an endovascular stentgraft.
\end{abstract}

Keywords: LVAD; Pseudoaneurysm; Endovascular stent

\section{Introduction}

Peudoaneurysms are rare complications related to left ventricular assist device (LVAD) implantation and mostly occur at the left ventricular apex, as well as the anastomosis of the outflow graft to the ascending aorta [1]. Beside anastomosis related complications and an erosion of the graft with a sternal wire they can occur due to an infection $[2,3]$. Nevertheless pseudoaneurysms represent a major complication potentially leading to significant morbidity and mortality and in most cases necessitating open-surgical management.

Here we report on a case of an infection-induced pseudoaneurysm in close proximity to the LAVD's outflow cannula which was treated successfully with an endovascular stentgraft.

\section{Case Report}

In March 2015, a 57-year-old male patient underwent implantation of a Heartmate II LVAD (Abbott, CA, USA) due to dilated cardiomyopathy with low output failure (ejection fraction 15\%). 10 months after implantation the device had to be replaced by a Heart Mate III (Abbott, CA, USA) due to driveline infection. After an uneventful course the patient was discharged in good condition and without residual signs of infection, to a rehabilitation hospital.

After 8 months the patient displayed a pulsatile painless swelling in the previous left anterolateral thoracotomy with no fever or clinical signs of infection. Lab values revealed an elevated C-reactive protein $(70.2 \mathrm{mg} / \mathrm{L})$ and a slightly lowered hemoglobin level $(9.4 \mathrm{~g} / \mathrm{dL})$ but a normal leukocytes count (4.6 Tsd/ $\mu \mathrm{L})$.

A computed tomography angiogram (CTA) showed $4 \mathrm{~cm}$ dehiscence just after the outflow graft from the LVAD take-off with an pseudoaneurysm measuring $7 \times 4 \mathrm{~cm}$ (Figure 1A).

Due to the patient's surgical history as well as the high risk of bleeding associated with another surgical approach, we decided to pursue an interventional/endovascular repair.

Based on the curved aortic anatomy and outflow graft shape, the short catheter shaft length and the small lumen of the supra-aortic branches, we chose an access through the ascending aorta via a ministernotomy. We also decided to use a Gore Excluder (WL Gore and Associates, Inc. Flagstaff, AZ) stentgraft (Gore C-TAG, $21 \mathrm{~mm}$ diameter $10 \mathrm{~cm}$ length) considering the matching size to the LVAD outflow Dacron-graft and the high flexibility of the delivery system.

A partial upper sternotomy was performed under general anesthesia. The ascending aorta was cannulated with a $5 \mathrm{Fr}$ introducer sheath. The patient received 5000 I.U. heparin to achieve an activated clotting time (ACT) of greater than $300 \mathrm{~s}$

A 0.035 ', $180 \mathrm{~cm}$ long Terumo Radiofocus ${ }^{\mathrm{TM}}$ guidwire (Terumo Europe N.V, Leuven, Belgium ) followed by a $5 \mathrm{Fr}, 65 \mathrm{~cm}$ long catheter (Cordis Tipperary, Ireland) was placed into the outflow graft. After exchanging the guide-wire to a Cook Lunderquist ${ }^{\mathrm{TM}}$ extra stiff Guidewire $0.035^{\prime}$ ', $180 \mathrm{~cm}$ long (Cook, Bloomington, USA), an 18 Fr Gore DrySeal $^{\mathrm{TM}}$ introducer sheath was inserted.

The pump was then turned off for approximately 2 min to push the guiding wire through the impella and to stabilize the stentgraft bearing catheter. Subsequently the aortic stent graft was directly aligned with the outflow graft and released. The LVAD was restarted to its original settings. A completion angiography was performed showing no further signs of aneurysm or extravasation/ bleeding. Finally all guiding materials were removed and the mini-incision of the sternum was closed with wires. The patient was extubated in the operation room and transferred to the intensive care unit for further treatment. After an uneventful $24 \mathrm{~h}$ time course he was transferred to the normal ward.

A follow up CT-scan one week after the procedure showed no signs of aneurysm, bleeding or residual haematoma (Figure 1B-1D). The post-operative course was uneventful and the patient is being

${ }^{*}$ Corresponding author: Saad Rustum, MD, Department of Cardiac, Thoracic Transplantation and Vascular Surgery, Hannover Medical School, Carl-NeubergStr 1, 30625 Hannover, Germany, Tel: +495115326589; Fax: +495115325867; E-mail: rustum.saad@mh-hannover.de

Received August 16, 2017; Accepted August 25, 2017; Published August 29, 2017

Citation: Rustum S, Schmitto J, Dogan G, Umminger J, Haverich A, et al. (2017) Successful Treatment of LVAD Patient with Infection-Induced Pseudoaneurysm using Endovascular Stent Graft. J Vasc Med Surg 5: 333. doi: 10.4172/23296925.1000333

Copyright: @ 2017 Rustum S, et al. This is an open-access article distributed unde the terms of the Creative Commons Attribution License, which permits unrestricted use, distribution, and reproduction in any medium, provided the original author and source are credited. 
Citation: Rustum S, Schmitto J, Dogan G, Umminger J, Haverich A, et al. (2017) Successful Treatment of LVAD Patient with Infection-Induced Pseudoaneurysm using Endovascular Stent Graft. J Vasc Med Surg 5: 333. doi: 10.4172/2329-6925.1000333

Page 2 of 2
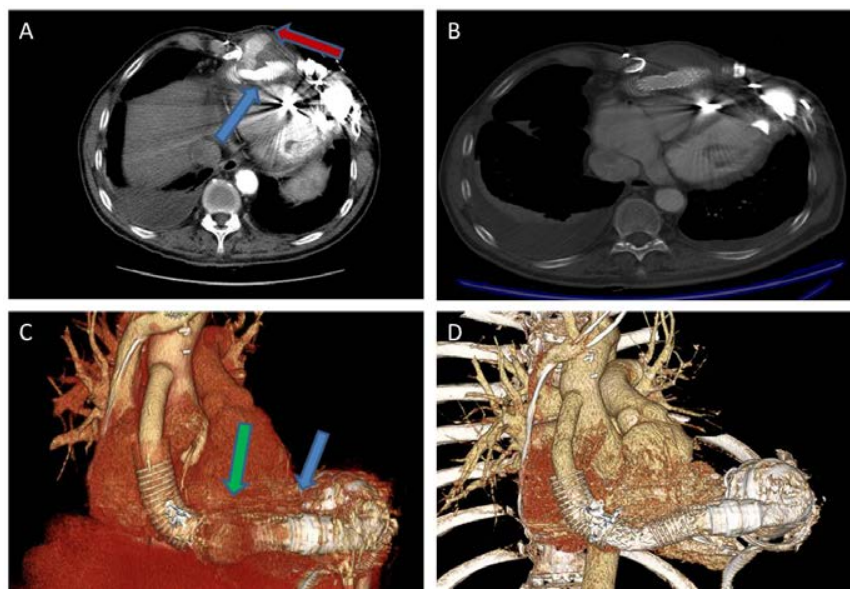

Figure 1: (A) Axial computed angiogram (CTA) showing pseudoaneurysm (red arrow) arising from the outflow graft of the left ventricular assist device (blue arrow). (B) Follow-up computed tomogaphy at 1 week showing well positioned stent without sign of pseudoaneurysm and no evidence of endoleak. (C) 3D reconstruction of an angio-CT scan demonstrating pseudoaneurysm (green arrow) with communication to the outflow graft of the left ventricular assist device (blue arrow). (D) 3D reconstruction of an angio-CT scan demonstrating well-positioned stent with no sign of pseudoaneurysm or endoleak.

considered for heart transplantation. The patient was discharged from hospital in good clinical condition with stable hemoglobin level. After a 6 month waiting period the patient was successfully transplanted.

\section{Discussion}

LVAD implantation is a standard therapy for the treatment of advanced and terminal heart failure. However, despite the positive effects, device-related severe and potentially life-threatening complications are frequently observed [3-6]. Most of these complications such as thrombosis, embolism, outflow graft/graft stenosis or obstruction, arteriobronchial fistulae and pseudoaneurysms are traditionally managed surgically through a sternotomy and often by pump exchange. Minimal invasive surgery has become a standard for the treatment of LVAD-associated complications such as pump thrombosis and driveline infection [7]. In contrast, reports on interventional repair of outflow graft because of bleeding or obstruction are sparse $[2,8,9]$.

A full sternotomy in a redo case is associated with an increased risk of bleeding because of accidental laceration of the outflow graft. Moreover there is a high probability of blood loss and the need for blood reserves. To prevent life-threatening complications as mentioned by high risk surgery and to avoid further adhesions to conserve the anatomical situation for heart transplantation we advocate endovascular repair. On the other hand, there is the risk of a difficult anatomy which complicates the endovascular procedure, and of damaging the pump by catheters or wires during the procedure. Overall the endovascular repair is the more cost effective procedure as compared to an expensive total pump exchange.

Other cases describe an approach via the supra-aortic branches, which was not suitable for the patient in this case. We performed a safe and straight-forward approach involving mini-sternotomy to insert the stent graft system into the LVAD's outflow graft.

\section{Conclusion}

Despite the development of newer and safer implantation strategies of LVADs, addressing LVAD-associated complications remains a challenge. Given the advantages and technical improvements of new generation endovascular tools in combination with safer minimalinvasive surgical approach, the endovascular approach presents a feasible, effective and alternative approach to managing LVAD-related complications, as opposed to invasive open surgery.

\section{References}

1. Maeda T, Tanoue $Y$, Nakashima A, Tominaga R (2010) Atypical presentation of an apical pseudoaneurysm in a patient on prolonged left ventricular mechanical support. Interact Cardiovasc Thorac Surg 10: 350-351.

2. Ganapathi AM, Andersen ND, Prastein DJ, Hashmi ZA, Rogers JG, et al (2013) Endovascular stent grafting of a left ventricular assist device outflow graft pseudoaneurysm. Circ Heart Fail. Jan 6: e16-e18.

3. Knosalla C, Weng Y, Buz S, Loebe M, Hetzer R (2000) Pseudoaneurysm of the outflow graft in a patient with Novacor N100 LVAD system. Ann Thorac Surg. May 69: 1594-1596.

4. Schmitto JD, Molitoris U, Haverich A, Strueber M (2012) Implantation of a centrifugal pump as a left ventricular assist device through a novel, minimized approach: upper hemisternotomy combined with anterolateral thoracotomy. J Thorac Cardiovasc Surg 143: 511-513.

5. Schmitto JD, Avsar M, Haverich A (2014) Increase in left ventricular assist device thrombosis. N Engl J Med 370: 1463-1464.

6. Starling RC, Moazami N, Silvestry SC, Ewald G, Rogers JG, et al. (2014) Unexpected abrupt increase in left ventricular assist device thrombosis. $\mathrm{N}$ Engl J Med 370: 33-40.

7. Rojas SV, Avsar M, Khalpey Z, Hanke JS, Haverich A, et al. (2014) Minimally invasive off-pump left ventricular assist device exchange: anterolateral thoracotomy. Artif Organs 38: 539-542.

8. Abraham J, Remick JD, Caulfield T, Puhlman M, Evenson K, et al. (2015) Left ventricular assist device outflow cannula obstruction treated with percutaneous endovascular stenting. Circ Heart Fail 8: 229-230.

9. Yanagida R, Kass R, Czer L, Khoynezhad A (2011) Endovascular repair of arterio-bronchial fistula of the outflow graft of HeartMate II left ventricular assist device. J Thorac Cardiovasc Surg 142: 710-711. 